\title{
MIF Proteins from Two Nematodes Recognize Sera of Onchocerciasis Patients and Stimulate Peripheral Blood Mononuclear Cells to Release TNF- $\alpha$ and iL-10 Cytokines
}

\author{
Boursou Djafsia ${ }^{1,}$, , Dieudonne Ndjonka ${ }^{1}$, Irene Ajonina-Ekoti ${ }^{2}$ \\ ${ }^{1}$ Department of Biological Sciences, Faculty of Science, University of Ngaoundere, Ngaoundere, Cameroon \\ ${ }^{2}$ Department of Molecular Physiology, University of Münster, Muenster, North Rhine-Westphalia, Germany
}

Email address:

vboursou1@gmail.com (B. Djafsia)

${ }^{*}$ Corresponding author

\section{To cite this article:}

Boursou Djafsia, Dieudonne Ndjonka, Irene Ajonina-Ekoti. MIF Proteins from Two Nematodes Recognize Sera of Onchocerciasis Patients and Stimulate Peripheral Blood Mononuclear Cells to Release TNF- $\alpha$ and iL-10 Cytokines. Journal of Diseases and Medicinal Plants.

Vol. 2, No. 2, 2016, pp. 8-13. doi: 10.11648/j.jdmp.20160202.11

Received: March 31, 2016; Accepted: April 13, 2016; Published: May 17, 2016

\begin{abstract}
Onchocerciasis is a human filarial disease which leads to blindness in its ultimate phase. The immunopathology of the disease categorize patients into two types regarding the symptoms of the sickness: the hypo-reactive (generalized) and the hyper-reactive (sowda) types. In vitro, the humoral response by sera from the two categories of patient to recombinant proteins from the human parasitic nematodes: Onchocerca volvulus and Strongyloides ratti and the free-living nematode: Caenorhabditis elegans showed differences in strength and specificity. Immune recognition of antigens from $O$. volvulus (extract, $O v$ mif 1 and $O v \mathrm{SOD}$ ) and $C$. elegans (mif proteins) were evaluated in a western blot and an indirect enzyme linkedimmunosorbent assay (ELISA) using 36 human sera. The response was determined as optical density (OD) read at $450 \mathrm{~nm}$ and an index value of recognition calculated. The rate of induction of pro and anti-inflammatory cytokines by the same proteins was assessed on peripheral blood mononuclear cells (PBMC). Variable amounts of recombinant proteins were incubated with PBMC at 5\% $\mathrm{CO}_{2}$ in RPMI 1640 medium supplemented with antibiotic, bovine serum albumin, HEPES. Generally, there was a stronger recongnition of $O$. volvulus antigens than $C$. elegans antigens. However, a comparison of the index values by ANOVA using Bonferroni post-test showed a significantly stronger recognition of $O$. volvulus antigens by sera from sowda patients than the recognition by sera from the generalized form when compared to $C$. elegans antigens. The sowda sera were shown therefore to be specific to $O$. volvulus antigens. The stimulation of PBMC by recombinant proteins from the two categories of nematode induced globally a higher anti-inflammatory (iL-10) cytokine release compared to the proinflammatory $(\mathrm{TNF} \alpha)$ cytokine. Again, there was a notable higher release of iL-10 and TNF- $\alpha$ by proteins from the parasitic nematodes compared to the free living nematode. The humoral immune response to a cocktail of recombinant proteins from $O$. volvulus and the bovine parasite Onchocerca ochengi should be evaluated.
\end{abstract}

Keywords: Onchocerca volvulus, Caenorhabditis elegans, Immunoglobulines, White Blood Cells, TNF $\alpha$

\section{Introduction}

Onchocerciasis is a filarial disease that causes a debilitating pathology which leads to blindness in its ultimate phase. This disease is transmitted by several species of Onchocerca gender [1]. In human, the disease is due to Onchocerca volvulus and transmitted by a black fly of gender Simulium which breeds in fast flowing rivers [2].
Onchocerciasis is endemic in 3 of the 6 continents of the world: Africa, America and Arabic peninsula in Asia with more than $90 \%$ of cases located in Africa [3]. Over 37 million people are infected worldwide with 500000 cases of blindness [4]. In Cameroon, more than 7 million people (nearly $50 \%$ of the entire population) are infected of which 32000 are cases of blindness (Kamgno, 2015 personal communication). The immunopathology of the human disease is characterized by a differential production of 
cytokines due to the immune system reaction and filarial modulation of the host. Following the reactivity, two categories of patients can be distinguished: generalized form and hyperreactive form «sowda». It has been shown that total immunoglobulin response is higher in the hyperreactive form than the generalized form [5]. The observed immune response is induced by the antigens originating from $O$. volvulus excretory and secretory (ES) proteins used by the worm to divert host immune responses [6]. Several groups of proteins are reported to interfere with the host immune system to allow infection to last for a long period of time in the body of the host [7]. These proteins play therefore a key role in the perpetuation of the pathology by diverting the adequate immune response to a favorable response. Sometimes reactivity to the infection can be noticed, especially in the case of dead microfilariae or an intrinsic reactivity of the host. This reactivity is the synergic effect of individual reactivities of immunoglobulin subtypes (IgG1, IgG2, IgG3, IgG4). Nevertheless, the most important effect in generalized and hyperreactive forms of human onchocerciasis is the reactivity of IgG4 subtype which is sometimes used for diagnosis in some kinds of filariasis [5, 8]. In revanche, some parasite antigens are able to induce a specific recognition to filariasis oriented towards the production of pro-inflammatory immune response. This aspect is investigated in the present study.

The aim of the current work was to examine the reactivity of sera from onchocerciasis infected human to recombinant proteins from the parasitic nematode $O$. volvulus, compared to the non parasitic nematode Caenorhabditis elegans and the specific pro and anti-inflammatory cytokines release induced by these proteins,. Firstly, an evaluation of the recognition of somatic $O$. volvulus extract by sera from generalized form of onchocerciasis was done using Western blot analysis. Secondly, the reactivity of sera from generalized and hyperreactive form of onchocerciasis to the parasitic nematodes $(O$. volvulus and Strongyloides ratti) and freeliving nematode (C. elegans) proteins was assessed by indirect ELISA. An early pro-inflammatory immune response could play a key role in the induction of immune response to filariasis invasion of the host preventing the establishment of the infection. To assess this, a quantification of pro and antiinflammatory cytokine release after stimulation of peripheral blood mononuclear cells (PBMC) from volunteers with the same proteins was done.

\section{Material and Methods}

Whole worm extract of $O$. volvulus was obtained after grinding and sonication on ice of female adults provided by the laboratory of molecular parasitology of the University of Hamburg.

\subsection{SDS PAGE and Immunoblot}

Antigens were run in polyacrylamide gel electrophoresis (SDS PAGE) following the principle of Laemmli (1970) with $5 \%$ collection gel and $12 \%$ stacking gel. The obtained gel was stained with rouge ponceau dye and distained with distilled water after confirmation of the presence of proteins. Gel proteins were transferred on nitrocellulose membrane by western blot with a transblot apparatus (Biostep/Germany). The nitrocellulose membrane was incubated in a 5\% PBSskim milk solution for $1 \mathrm{~h}$ at room temperature and washed 2 times with PBS. The membrane was collected and cut into slices corresponding to the lanes defined by the transit of the sample from the top to the bottom of the SDS PAGE. Each slice was incubated over night at $4^{\circ} \mathrm{C}$ with the corresponding human serum $(1 / 250$ in $0,5 \%$ PBS-skim milk). After a washing step, membranes were incubated in a goat antihuman IgG peroxidase conjugated (Sigma) $1 / 1000$ for $2 \mathrm{~h}$ followed by a washing step ( 3 times). The revelation was made with $10 \%$ 4-chloro-1-naphtol $+20 \mu \mathrm{l}$ of $\mathrm{H}_{2} \mathrm{O}_{2}$ and the membrane washed with PBS, distilled water and dried under pressure within Wattman papers. The dried membrane slices were join together and scanned.

\subsection{Enzyme Linked Immunosorbent Assay (ELISA)}

Semi-quantitative analysis of serum IgG antibody level was performed by ELISA to find antibodies end point as previously described by Mpagi et al. (2000) [9] with modifications. $O$. volvulus extract and recombinants proteins were coated at $200 \mathrm{ng}$ per well using 96 wells polystyrene plate (Nunc, Roskilde, Denmark) in carbonate buffer ( $\mathrm{pH} 9.6$ ) and incubated overnight at $4^{\circ} \mathrm{C}$. The incubation was made with $O$. volvulus extract and recombinant proteins such as macrophage migration inhibitory factor (MIF) from $O$. volvulus [10], C. elegans and S. ratti. 24 hours later, the plate was washed 4 times with PBS $+0.05 \%$ Tween 20 . After removing unbound proteins, the plate was blocked with 200 $\mu 1$ of $5 \%$ bovine serum albumin (BSA) per well for 2 hours at $37^{\circ} \mathrm{C}$. The BSA excess was then washed 4 times and the plate incubated for another 2 hours at $37^{\circ} \mathrm{C}$ with human sera $(1 / 2000,1 /, 000$ and $1 / 32000)$ diluted in $200 \mu \mathrm{l}$ PBS- $0.5 \%$ BSA. The non-specifically bound antibodies were washed and the plate incubated with $100 \mu$ of $1 / 1000$ horseradish goat anti-human (Roche, Mannheim/Germany) for an hour at $37^{\circ} \mathrm{C}$. The anti-human $\operatorname{IgG}$ excess was removed by another washing step. The detection was done using $100 \mu \mathrm{l}$ of tetramethyl benzidine substrate (TMB) in the dark for $5 \mathrm{~min}$ and the reaction stopped with $0.02 \% \quad \mathrm{H}_{2} \mathrm{SO}_{4}$ at room temperature. The optical density (OD) was measured at 405 nm using ELISA reader (Dynatech).

\subsection{Blood Cells Harvest and Culture}

Peripheral blood mononuclear cells (PBMC) were collected following the modified procedure of Brattig et al. (2001) [11]. Venous blood was collected from healthy volunteers in a $10 \mathrm{ml}$ collection tubes containing $0.106 \mathrm{~mol} / 1$ of trisodium citrate and $1 \mathrm{ml}$ of citrate solution (Sarstedt, Germany). In $5 \mathrm{ml}$ of sterile PBS, an equal volume of blood was added and was mixed by hand gently for $2 \mathrm{~min}$. In a different reaction tube containing about $3 \mathrm{ml}$ of gradient solution (lymphoflot biotest/Germany) $6 \mathrm{ml}$ of Blood-PBS 
mixture was gently added and the tube centrifuged at 1700 $\mathrm{rpm}$ for $10 \mathrm{~min}$. the first cellular layer was taken with a micropipette. The collected cells were put in culture in $5 \%$ $\mathrm{CO}_{2}$ incubator in RPMI 1640 supplemented with antibiotics (100 units/ml penicillin and $100 \mu \mathrm{g} / \mathrm{ml}$ streptomycin), $30 \mathrm{mM}$ HEPES and $10 \%$ bovine serum albumin at $37^{\circ} \mathrm{C}$. The cultured cells were split at their exponential phase of growth and transferred into 24 well plates (Nunc Roskilde, Denmark). The cells (500 000 /well) were stimulated using proteins as antigens with hemaglutinin (PHA) and lipopolysaccharide (LPS) as positive control. After $48 \mathrm{~h}$ of incubation, the culture media were collected, centrifuged (12 $000 \mathrm{rpm}$ for $15 \mathrm{~min}$ ) and cytokine quantified by ELISA (eBioscience kit) at $450 \mathrm{~nm}$.

\subsection{Data Analysis}

Data were expressed as OD x 1000 for cytokine quantification ELISA. Data analysis and figure plotting were performed using graph pad prism 5.0. Two way analysis of variance (ANOVA) followed by the Bonferroni test were used to compare groups of antigens and cytokines production. For IgG recognition of proteins OD values were transformed using a regression formula, $\mathrm{y}=\mathrm{ax}+\mathrm{b}$ where $\mathrm{x}=$ index, $\mathrm{y}=$ ODs and $\mathrm{a}=$ the slope calculated with the standard dilution of $1 / 500,1 / 1000$ and $1 / 2000$ and $b=y_{0}$. OD values were presented as mean $+/$ - standard error with values $<0.1$ being discarded and $\mathrm{p}<0.05$ was considered significant. If not stated otherwise, all chemicals were purchased from SigmaAldrich (Deisenhofen, Germany).

\section{Results}

\subsection{Immunoblot and ELISA}

The pattern of worm proteins was recognized by the total IgG of human sera. Naive sera recognized very slightly the somatic extract compared to the infected ones (figure 1). Several bands can clearly be identified especially low molecular weight bands at 15, 16, 25, 30 and $50 \mathrm{kD}$ approximately.

Recombinant proteins from nematodes (parasites and freeliving) were recognized by immunoglobulins of infected human sera from both disease forms (generalized and hyperreactive). The recognition of recombinant proteins of parasitic worms was generally stronger than those of the freeliving worm. The index value is shown for each recombinant protein from O. volvulus (mif 1, SOD) and Strongyloides ratti (mif) and C. elegans (mif 1, 2 and 4) (figure 2). Generally, the recognition of antigens of the parasite nematode (figure 2 a) by sera of sowda form seems to be higher than the recognition of sera of the generalized form with a maximum of index value at 6602 and a minimum of 1639. The proteins from free-living nematode (C. elegans) displayed a slightly higher recognition by sera from generalized form.

A two-way analysis of variance (ANOVA) showed that, the different types of sera did not have the same effect on the recombinant proteins of parasitic nematodes,. In addition, neither the type of sera nor the antigens affect significantly the obtained results in the same analysis. The Bonferroni post test following the two-way ANOVA allowed drawing the table 1 and 2. Both tables (table 1 and table 2) display the differences between the reactivity of each antigen to naïve against sowda sera and naïve sera against generalized form sera respectively for parasitic and free-living nematodes. The table 1 displays the difference between $O$. volvulus female extract and recombinant proteins from the same parasite and table 2 shows the statistic differences between $O$. volvulus extract and $C$. elegans recombinant mif proteins in a Bonferroni comparison test.

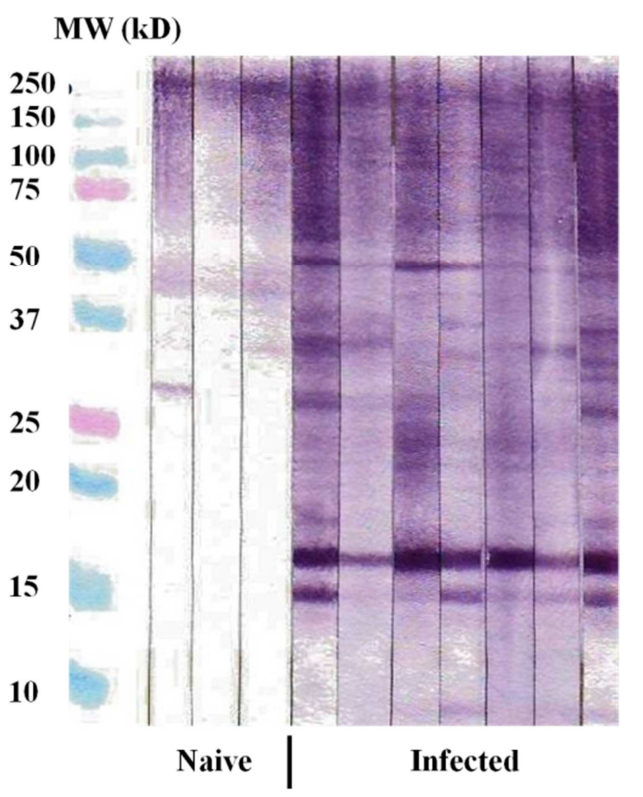

Figure 1. Immunobloting of O. volvulus whole extract proteins.

Table 1. Immuno-reactivity results after Bonferroni post test comparing naïve sera against sowda groups for recombinant proteins from Onchocerca volvulus and Strongyloides ratti.

\begin{tabular}{lllll}
\hline Antigens & Difference & t & P value & Summary \\
\hline Extract & 3250 & 10,5 & $\mathrm{P}<0.001$ & $* * *$ \\
Ov mif 1 & 927,4 & 2,9 & $\mathrm{P}<0.05$ & $*$ \\
Ov SOD & 486,4 & 1,6 & $\mathrm{P}>0.05$ & $\mathrm{~ns}$ \\
Sr mif & 911,80 & 3,01 & $\mathrm{P}<0.05$ & $*$ \\
\hline
\end{tabular}

ns: non significative difference

Table 2. Immuno-reactivity results after Bonferroni post test comparing naïve sera against sowda groups for recombinant proteins from Caenorhabditis elegans.

\begin{tabular}{lllll}
\hline Antigens & Difference & t & P value & Summary \\
\hline Extract & 6188 & 14 & $\mathrm{P}<0.001$ & $* * *$ \\
Ce mif 1 & 1154 & 3 & $\mathrm{P}<0.05$ & $*$ \\
Ce mif 2 & 537,9 & 1,2 & $\mathrm{P}>0.05$ & $\mathrm{~ns}$ \\
Ce mif 4 & 1198 & 3 & $\mathrm{P}<0.05$ & $*$ \\
\hline
\end{tabular}

ns: non significative difference 


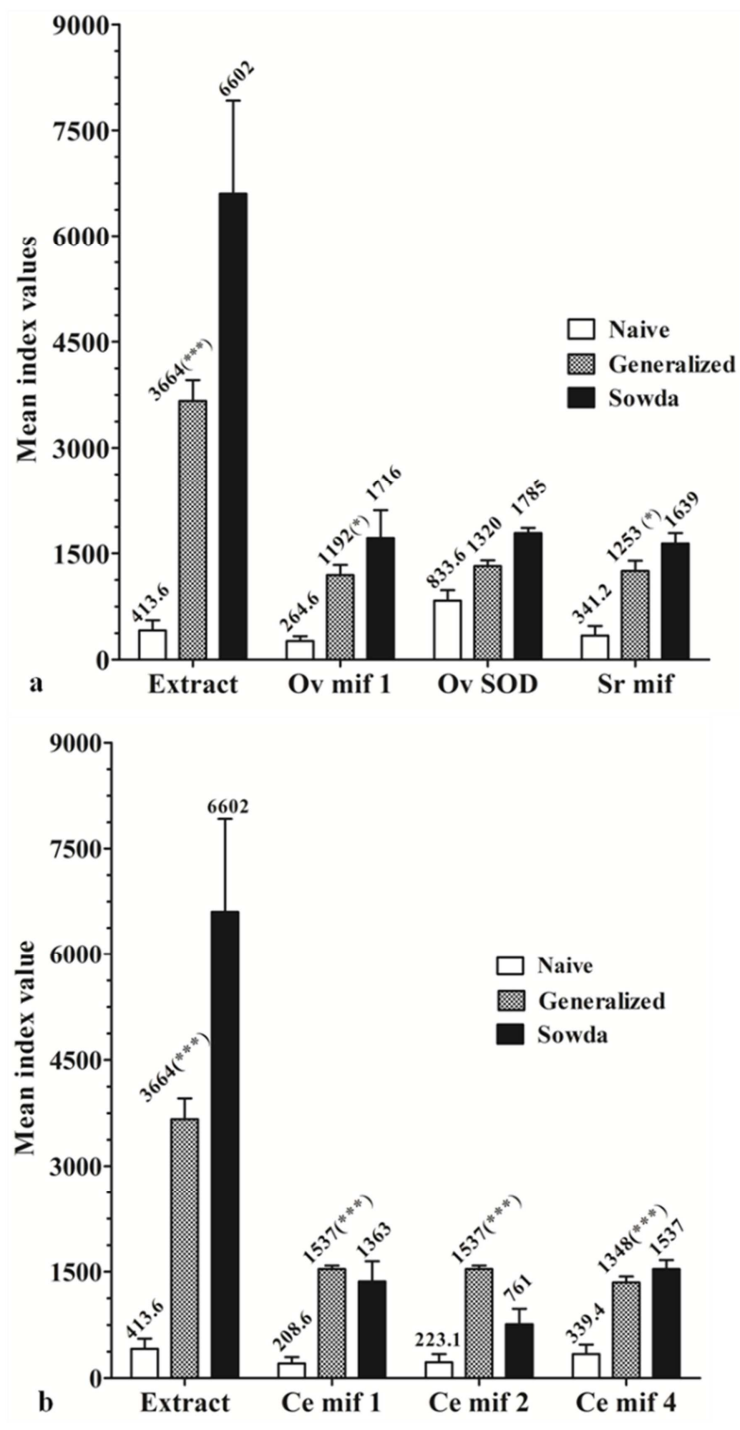

Figure 2. Immune recognition of proteins of (a) parasitic Onchocerca volvulus and Strongyloides ratti and (b) free-living Caenorhabditis elegans nematodes by onchocerciasis infected human sera from generalized and hyperreactive form.

\subsection{Cytokine Release of PBMC}

Figure 3 displays the optical density (OD) value representing the release of cytokines (TNF $-\alpha$ and iL-10) after stimulation with recombinant proteins from the parasitic nematode $O$. volvulus and the free-living nematode $C$. elegans. Generally, an elevated quantity of iL-10 compared to the TNF $\alpha$ is noticeable for recombinant proteins and positive controls (PHA and LPS). Increased amount of iL-10 is related to a humoral downregulation of inflammation phenomenon and decreased amounts means the reverse effect. Thus a high amount of TNF- $\alpha$ is a sign of inflammation and immune reaction of the host to a pathogen. The recombinant proteins induced release of iL-10 for $O$. volvulus and $C$. elegans. This is not the case with the pro-inflammatory cytokine TNF- $\alpha$ which shows a relatively low reactivity with $C$. elegans mif proteins compared to $O$. volvulus proteins.

For $O$. volvulus proteins, the amount used for stimulation is proportional to the quantity of TNF- $\alpha$ and iL-10 released except for iL-10 release induced by $O v$ mif 1 . Thus, for an amount of $5 \mu \mathrm{g}$ the response of cells in term of TNF- $\alpha$ release is lower than $25 \mu \mathrm{g}$ of LPS of $O v \mathrm{SOD}$ (figure 3). A reverse effect is observed following the stimulation with $C$. elegans proteins except with Cemif 4 which induced TNF- $\alpha$ proportionally to the amount used for stimulation as observed with most of the $O$. volvulus proteins.

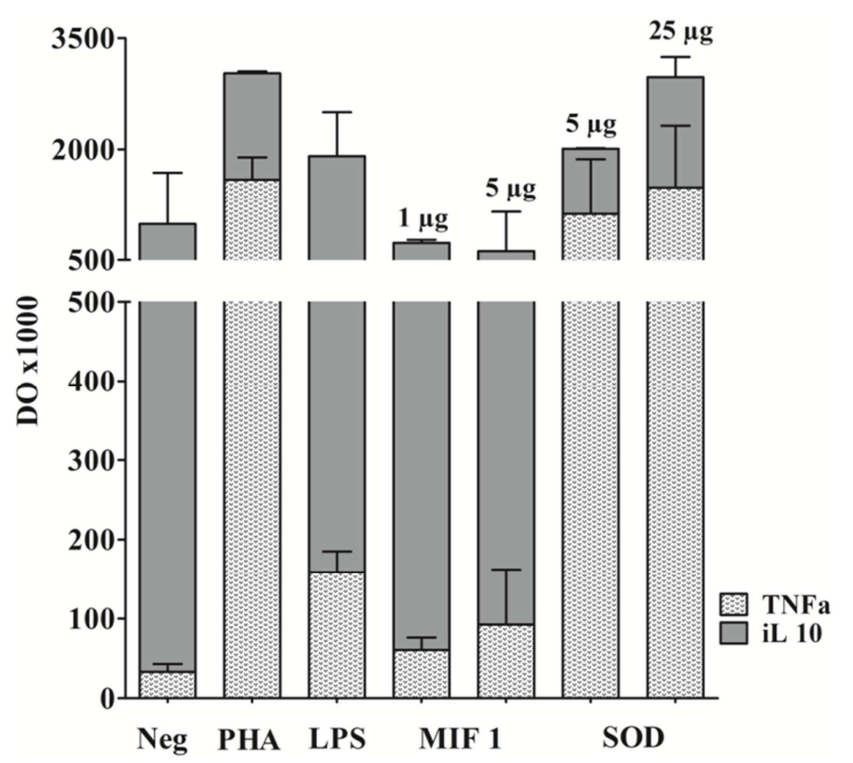

(a)

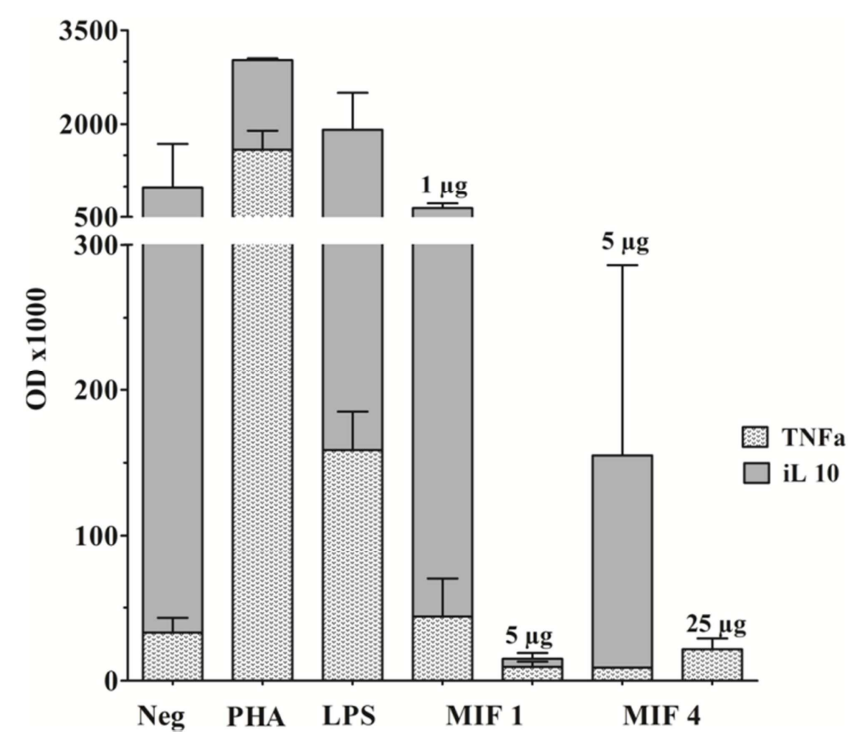

(b)

Figure 3. Tumor necrosis factor alpha $(T N F \alpha)$ and interleukine 10 release of human peripheral blood mononuclear cells from healthy patients after stimulation with recombinant proteins from (a) parasitic and (b) free-living nematodes

\section{Discussion}

The recognition of proteins from $O$. volvulus by onchocerciasis patients is an argument in favor of the specificity of IgG from the generalized form of onchocerciasis. The observed recognition is due to the 
presence of antigens in the whole worm extract specific to the immunoglobulins produced during the disease pathology in humans [12]. In the case of our study, the protein mixture shown in western blot (figure 1) is recognized strongly by sera from onchocerciasis patients. The density of recognized bands justify why a cocktail of proteins is well recognized by the sera used. In fact, the recognition could be a cumulative effect of the individual recognition of each of the affinity proteins present in the protein cocktail (extract) [13].

There was no significant difference in the immune recognition of proteins from $O$. volvulus by both categories of sera $(\mathrm{p}<0.05)$. Nevertheless, most of the antigens were strongly recognized by sowda patients sera than naïve sera (table 1). There was no significant difference in the immune recognition of proteins from $C$. elegans by both categories of sera, although the response was relatively higher in the generalized form than in the sowda form except for Cemif2 (figure $2 \mathrm{~b}$ ) . The observed results show that immunoglobulins produced by sowda patients are more specific to $O$. volvulus proteins and can therefore, be used as a diagnostic tool in human onchocerciasis [14]. This corresponds with previous studies that reported that, IgG4 immunoglobulin subtype in sowda patients was specific to Onchocerca antigens [9].

The anti-inflammatory cytokine (iL-10) release was shown to be more abundant than pro-inflammatory cytokine (TNF- $\alpha$ ) for the antigens used (C. elegans, $O$. volvulus and controls). This is in line with other studies that reported the same downregulatory effects with cystatin a secretory protein from O. volvulus and Acanthocheilonema vitae [7; 15]. The high release of TNF- $\alpha$ noticed in the present study confirms a similar observation in an early filarial infection with an increased secretion of the pro-inflammatory cytokines TNF- $\alpha$ and iL-8 [16, 17, 18]. Generally, an early onchocerciasis infection is characterized by the release of pro-inflammatory cytokine and a moderate cellular response. Macrophage migration inhibitory factor (MIF) proteins from human are reported to induce inflammatory cytokine release during inflammation process [19]. In our case, the isolated reactivity of MIF proteins from $O$. volvulus, $S$. ratti and $C$. elegans showed a proeminent anti-inflammatory cytokine release. This observation could be explained by the fact that the MIF protein present in the whole protein cocktail (ES proteins) induced the release of anti- and pro-inflammatory cytokines such as iL-10 and TNF- $\alpha$. On the other hand, OvSOD a characteristic filarial ES proteins has been reported to be specifically targeted by humoral immune response of Onchocerca infected patients [10, 20].

\section{Conclusion}

In conclusion, macrophage migration inhibitory factor protein from $C$. elegans, $O$. volvulus and $S$. ratti were targeted by humoral response of onchocerciasis patients as well as $O$. volvulus extract. Sera of the sowda form of onchocerciasis strongly recognized with the highest specificity antigens from $O$. volvulus origin compared to sera of the generalized form. The same antigens elicited predominantly anti-inflammatory cytokine (iL-10) with a little difference in the strength of reactivity between parasitic worm and free-living worm proteins. Additional information on the cytokine profile during onchocerciasis infection could be obtained by evaluating humoral response and immune recognition of a cocktail of $O$. volvulus ES proteins and even the cross reactivity with his closest relative Onchocerca ochengi proteins which will therefore reinforce the present data.

\section{Authorship Contribution}

All authors contributed to the designing, preparation, editing, and final review of the manuscript.

\section{Acknowledgement}

This work is a part of the $\mathrm{PhD}$ thesis of B. Djafsia. This research was supported by the fellowship (equipment supply) of the Alexander von Humboldt Foundation (AvH) to Pr. D. Ndjonka.

\section{References}

[1] Achukwi MD, Harnett W, Renz A., 2000. Onchocerca ochengi transmission dynamics and the correlation of $O$. ochengi microfilaria density in cattle with the transmission potential. Vet. Res. 31 (6): 11

[2] Basáñez M-G, Pion SDS, Churcher TS, Breitling LP, Little MP, Boussinesq M., 2006. River Blindness: A Success Story under Threat? PLoS Med. 3.

[3] Turner JD, Tendongfor N, Esum M, Johnston KL, Langley RS, Ford L, Faragher B, Specht S, Mand S, Hoerauf A, Enyong P, Wanji S, Taylor MJ., 2010. Macrofilaricidal Activity after Doxycycline Only Treatment of Onchocerca volvulus in an Area of Loa loa Co-Endemicity: A Randomized Controlled Trial. PLoS Negl. Trop. Dis. 4, e660.

[4] Tropical disease research (TDR). 2005. Onchocerciasis. Seventeenth program report: progress 2003-2004. 44-49, TDR/GEN/05.1.

[5] Dafa'alla TH, Ghalib HW, Abdelmageed A, Williams JF., 1992. The profile of IgG and IgG subclasses of onchocerciasis patients. Clin. Exp. Immunol. 88: 258-263.

[6] Hewitson JP, Grainger JR, Maizels RM., 2009. Helminth immunoregulation: the role of parasite secreted proteins in modulating host immunity. Mol. Biochem. Parasitol. 167: 111 .

[7] Maizels RM, Yazdanbakhsh M., 2003. Immune Regulation by helminth parasites: cellular and molecular mechanisms. Nat. Rev. Immunol. 3: 733-744.

[8] Rahmah N, Khairul Anuar A, Tengku Ariff RH, Zurainee MN, Noor A'shikin A, Fadzillah A, Maimunah A, Ashraful Haq J., 1998. Use of antifilarial IgG4-ELISA to detect Brugia malayi infection in an endemic area of Malaysia. Trop. Med. Inter. Hlth. 3: 184-188. 
[9] Mpagi JL, Büttner DW, Tischendorf FW, Erttmann KD, Brattig NW., 2000. Humoral responses to a secretory Onchocerca volvulus protein: differences in the pattern of antibody isotypes to recombinant Ov20/OvS1 in generalized and hyperreactive onchocerciasis. Parasit. Immunol. 22: 455460 .

[10] Ajonina-Ekoti I, Ndjonka D, Tanyi MK, Wilbertz M, Younis AE, Boursou D, Kurosinski MA, Eberle R, Lüersen K, Perbandt M, Breloer M, Brattig NW, Liebau E., 2012. Functional characterization and immune recognition of the extracellular superoxide dismutase from the human pathogenic parasite Onchocerca volvulus (OvEC-SOD). Acta Trop. 124: 15-26.

[11] Brattig NW, Büttner DW, Hoerauf A., 2001. Neutrophil accumulation around Onchocerca worms and chemotaxis of neutrophils are dependent on Wolbachia endobacteria. Microbes Infect. 3: 439-446.

[12] Ho O, Edeghere H, Galadima M, Odama LD and Engelbrecht F., 2013. Antibody responses to a recombinant Onchocerca volvulus antigen (Ov1.9) by onchocerciasis patients before and two months after ivermectin treatment. Res. J. Biotech. 2 (1): 8-12.

[13] Djafsia B, Ndjonka D, Dikti JV, van Hoorn S, Manchang K, Brattig N, Liebau E., 2015. Immune recognition of excretory and secretory products of the filarial nematode Onchocerca ochengi in cattle and human sera. J. Helminthol. 1-9.

[14] Chandrashekar R, Masood K, Alvarez RM, Ogunrinade AF,
Lujan R, Richards FO, Weil GJ., 1991. Molecular cloning and characterization of recombinant parasite antigens for immunodiagnosis of onchocerciasis. J. Clin. Invest. 88: 14601466.

[15] Schierack P, Lucius R, Sonnenburg B, Schilling K, Hartmann S., 2003. Parasite-Specific Immunomodulatory Functions of Filarial Cystatin. Infect. Immun. 71: 2422-2429.

[16] Schönemeyer A, Lucius R, Sonnenburg B, Brattig N, Sabat R, Schilling K, Bradley J, Hartmann S., 2001. Modulation of Human $\mathrm{T}$ Cell Responses and Macrophage Functions by Onchocystatin, a Secreted Protein of the Filarial Nematode Onchocerca volvulus. J. Immunol. 167: 3207-3215.

[17] Brattig NW., 2004. Pathogenesis and host responses in human onchocerciasis: impact of Onchocerca filariae and Wolbachia endobacteria. Microbes Infect. 6: 113-128.

[18] Maizels RM, Pearce EJ, Artis D, Yazdanbakhsh M, Wynn TA., 2009. Regulation of pathogenesis and immunity in helminth infections. J. Exp. Med. 206: 2059-2066.

[19] Zang X, Taylor P, Wang JM, Meyer DJ, Scott AL, Walkinshaw MD, Maizels RM., 2002. Homologues of Human Macrophage Migration Inhibitory Factor from a Parasitic Nematode gene cloning, protein activity, and crystal structure. J. Biol. Chem. 277: 44261-44267.

[20] Cho MK, Ahn SC, Kim D-H, Yu HS., 2010. Parasite excretory-secretory proteins elicit TRIF dependent CXCL1 and IL-6 mediated allergic inflammation. Parasit. Immunol. 32: $354-360$. 